\title{
Assessment of Relationships Between Site-Specific Variables
}

\author{
P.P. Hujoel, *W.J. Loesche, ${ }^{\prime}$ and T.A. DeRouen ${ }^{*}$
}

THE ASSESSMENT OF RELATIONSHIPS between site-specific variables has been a matter of controversy because of the claim that periodontal sites within individuals can be used as independent observations in statistical models. One problem with this approach is the unreliability of the calculated Type I and Type II error rates. Another problem is that such inappropriate analysis may prohibit a correct assessment of causal relationships between site-specific variables. The host-factor can act as an effect modifier and modulate the magnitude of the site-specific effects and/or the host-factor can act as a confounder by superimposing a patient-effect on the studied site-specific effects leading to bias. As a result, site-specific biological mechanisms of disease progression may be misinterpreted. Sites can be used as the experimental unit of analysis, but the sampling design from which the site-specific data originated should not be ignored. $J$ Periodontol 1990;61:368-372.

Key Words: Models, statistical; host-factors; error rates; site-specific effects.

Several investigators suggest that averaging site-specific information within patients obscures the clinical and microbiological reality of periodontal disease and accordingly recommend that data analysis should occur on a site-specific basis. ${ }^{1-3}$ However, the practical realization of this approach has been complicated by the fact that inappropriate statistical techniques were used. ${ }^{4,5}$ Sites within a patient cannot be assumed independent as they are inherently correlated. ${ }^{6}$ For instance, periods of disease activity in a patient may be correlated due to a genetic and/or systemic predisposition, or treatment responses may be correlated due to the presence of an immunological response. It has been reported that ignoring this dependence is likely to increase the probability of a Type I error: a conclusion of significant differences when there are no 'true' differences present. . $^{7,8}$

Since causal inferences are often based on a probabilistic framework, statistical models built on incorrect assumptions can also affect the causal assessment of the investigated associations. If site-specific data are pooled, ignoring the patient as a covariate, inconsistent biological phenomena across patients can remain undetected, the strength of an association can be over- or underestimated, and the specificity of an association can be obscured due to the pooling of heterogeneous data. Although these three factors, strength, consistency, and, specificity, are not necessary criteria to justify inferring causal relationships, they have been rec-

*Department of Biostatistics, University of Michigan, Ann Arbor, MI. $\dagger$ Department of Biological and Material Sciences.

$\ddagger$ Department of Biostatistics and Department of Dental Public Health Sciences, University of Washington, Seattle, WA. ommended as the most useful criteria for separating association from causation..$^{9,10}$ If they are misrepresented and/ or ignored as a result of inappropriate analyses, the assessment of causality may be endangered, since it cannot be evaluated whether characteristics associated with the patient factor acted as a confounding factor (defined as a distortion of the effect measure), or as an effect modifier (defined as different values of the effect measure at different levels of patient characteristics).

The purpose of this paper is to show that the assessment of the relationships between site-specific variables requires adjustments for the patient as an additional source of variation, not only for statistical reasons, but also for inferring causal relationships. Common important criteria such as strength, consistency, and specificity, and related concepts such as effect-modification and confounding used for assessing the causality of a relationship between site-specific variables may become obscured with inappropriate analyses. Some of the issues are illustrated with an example.

\section{GENERAL PROCEDURE}

The search for etiological agents in periodontal disease is often focused on attempting to establish causal relationships between site-specific variables. For instance, studies which try to establish a relationship between a putative pathological organism (a site-specific factor) and disease activity (a site-specific response) would fall under this category. Most statistical methods appropriate for analyzing these types of data address to a certain extent the following questions: 1) are the observed site-specific associations consistent (homogeneous) across patients? 2) what is the strength of the 
association? and 3) is the association significant? These different steps will be discussed in the following paragraphs.

\section{Consistency}

Commonly, the first step consists of investigating whether the (site-specific) associations, estimated at the level of the individual patient, are consistent across patients. The concept of this testing for consistency (or homogeneity) of multiple within-patient (e.g., site-specific) responses across patients is relatively unique to dentistry. Medical investigations, especially when related to the inference of causality, commonly investigate the consistency of patient-specific responses across different groups of patients or populations. However, the principles involved for both medical or dental studies are similar. When patients are the elementary unit of analysis, the consistency is evaluated across different groups of patients or populations. When sites are the elementary unit of analyses, the consistency of the responses should be evaluated across patients.

Two general approaches can be used to analyze the data and to evaluate the consistency of the associations across patients: stratified analyses and/or mathematical modeling techniques. Stratified analyses offer the advantage of being relatively simple to perform and easy to interpret. Also, a limited number of assumptions are necessary to perform the statistical analysis. Inferences of the results of stratified analyses are usually restricted to only those patients of the sample. Mathematical modeling offers the advantage of a more parsimonious presentation of the data and the possibility to make inferences to the population; however, mathematical models, such as random effects models, make distributional assumptions about patient effects (e.g., that patient effects are normally distributed with mean zero). Care should be taken when these models are used to insure that the assumptions hold approximately true for the data at hand; violation of this assumption may lead to misleading conclusions when the patient effects are heterogeneously (inconsistently) distributed.

Stratified analyses may offer the opportunity to use formal hypothesis tests to investigate the consistency (homogeneity) of the observed associations. However, it is generally accepted that the determination of (in)consistency of associations cannot be evaluated by merely testing the hypothesis of homogeneity at some preset significance level. Important factors such as the number of patients and the number of sites sampled per patient need to be considered when evaluating the $P$-value from a test of homogeneity. Apart from statistical considerations, the choice of an appropriate Type-I error rate needs to be determined by how much heterogeneity the investigator is willing to accept. In the clinical sciences it can be too much to expect constant effects across all patients or groups of patients. What is one person's homogeneity may be another person's heterogeneity, and vice-versa. ${ }^{11}$ In general, conclusions with regard to homogeneity should be made with great care, and the primary objective should not be to show that associations are inconsistent across patients, but rather to evaluate to what extent (and on what scale) associations may be considered homogeneous across patients. ${ }^{11}$

Dispite this subjectivity involved in investigating the (in) consistent nature of the associations with data, the evaluation process and the resulting conclusions can be important; it can convey significant biological information to the investigator, generate new hypothesis, suggest the presence of additional causal mechanisms, and dictate the direction of further statistical analysis. ${ }^{12-14}$ Obviously, the finding that associations are consistent across patients provides one supportive argument in favor of the studied causality of the association and may permit the use of more sophisticated mathematical models.

The finding that the associations are inconsistent results in a more challenging problem. Sometimes obvious inconsistent responses can be separated and further analysis performed on homogeneous subgroups. A thorough screening of patient-specific covariates such as sex, immunological criteria, the use of certain medications, etc. and their coincidence with the subgroups may be of significant value in understanding and interpreting the results of the study. A certain amount of subjective judgment may be required to identify and describe the (homogeneous) subgroups and any resulting findings should not be interpreted as conclusions, but rather as an hypothesis-generating part of the study. In other instances, the characterization of different homogeneous subgroups and their possible relationships to patient-specific characteristics may be impossible.

\section{Strength of the Association}

After this important first step of investigating the consistency of the observed association across patients, the second step is to investigate the strength of the association by establishing the direction and the magnitude of the relationship. Depending on the study design and the categorical or continuous nature of the data, several measures of association are available for use: odd ratios, relative risk estimates, average slope estimates, etc. When no confounding patient effects are present, the results of analysis which ignore the patient as a covariate (i.e., assume independence of sites), and analyses which take into account the patient (stratified analyses or mathematical modeling techniques) will result in similar estimates of strength of association. However, when confounding patient-effects are present, differences in the magnitude as well as the direction of the estimates may result between adjusted and unadjusted analyses.

\section{Significance of the Association}

As a final step in the analysis, the relationship can be tested at a preset significance level. This is also sometimes called a test for main effects: it will test the significance of the relationship between the site-specific variables. This aspect of the analysis has been discussed in several reports. ${ }^{4-7}$

The circumstances under which these statistical topics 
become important for the biological interpretation of the data are twofold: the patient factor can act as an effect modifier and/or a confounding factor. An effect modifier refers to a change of the effect of a site-specific exposure variable according to a patient-specific characteristic. For example, different levels of neutrophil status could possibly act as an effect modifier upon the association between sitespecific bacterial pathogenicity and disease progression. A confounding patient-effect refers to a super-imposition of the effect of a patient-specific factor on the estimated relationship between two site-specific variables. For example, pregnancy could act as a confounding variable in studies where the site-specific effect of a specific bacterial exposure variable on the presence of bleeding is evaluated. (Pregnancy may be a risk factor for gingival bleeding due to hormonal changes and its interaction with other bacterial exposures and may, at the same time, be related to the exposure variable under study.) These two biological concepts, effect modification and confounding due to patient effects, may be difficult to evaluate with analyses which ignore the sampling design from which the site-specific data originated. Stratified analyses as suggested above may often provide some guidance in the evaluation of the patienteffects (i.e., effect modification could present itself as significant heterogeneity of the observed site-specific associations across patients, and confounding could be detected due to large differences between the unadjusted and adjusted site-specific associations). In some instances, patient effects could act as effect modifiers and confounding factors simultaneously. A more detailed discussion of these issues can be found in several reference works. ${ }^{10,14,16}$

\section{AN EXAMPLE}

As a numerical example, consider the following hypothetical data from a case-control study: an investigator is interested in assessing the hypothesis that site-specific exposure to factor A causes a specific periodontal disease condition; 155 sites with a certain disease characteristic (cases) and 164 sites without the condition (controls) are selected from 8 patients and classified according to their exposure to factor A. The data are presented in Table 1 .

Two fundamentally different approaches are possible for this dataset: 1) one (inappropriate) approach would be to assume that sites are independently distributed and that the sampling design from which the data originated are irrelevant to the statistical model employed; and 2) another approach would be to stratify the data based on the patients, or, to use a more sophisticated mathematical modeling approach where certain assumptions will need to be made about the distribution of the patient effect (i.e., normally distributed). For both approaches the hypothesis of interest is that the presence of factor $A$ is associated with the disease characteristic.

As a first approach, it will be assumed that the sampling design from which the site-specific data originated cannot be ignored and that the patient factor needs to be adjusted
Table 1. Hypothetical Data From a Case Control Study

\begin{tabular}{lccrrrr}
\hline Patient & Sex & $\mathrm{D}^{-} / \mathrm{E}^{-}$ & $\mathrm{D}^{+} / \mathrm{E}^{-}$ & $\mathrm{D}^{-} / \mathrm{E}^{+}$ & $\mathrm{D}^{+} / \mathrm{E}^{+}$ & Odds ratio \\
\hline 1 & $\mathrm{~F}$ & 7 & 9 & 1 & 22 & 17.11 \\
2 & $\mathrm{~F}$ & 6 & 14 & 1 & 23 & 9.86 \\
3 & $\mathrm{~F}$ & 9 & 12 & 2 & 20 & 7.50 \\
4 & $\mathrm{~F}$ & 8 & 13 & 1 & 21 & 12.92 \\
5 & $\mathrm{M}$ & 7 & 1 & 18 & 1 & 0.39 \\
6 & $\mathrm{M}$ & 4 & 2 & 19 & 3 & 0.32 \\
7 & $\mathrm{M}$ & 9 & 3 & 33 & 5 & 0.45 \\
8 & $\mathrm{M}$ & 10 & 3 & 29 & 3 & 0.34 \\
Total & & 60 & 57 & 104 & 98 & $0.99^{*}$ \\
\hline
\end{tabular}

$\mathrm{D}^{+}$and $\mathrm{D}^{-}$represent respectively the presence or absence of the disease factor of interest. $\mathrm{E}^{+}$and $\mathrm{E}^{-}$represent respectively the presence and $a b-$ sence of a suspected etiological cause, factor A. Site-specific analyses which ignore the patient as a covariate would only use the total counts in their analyses and yield the corresponding unadjusted odd's ratio $\left({ }^{*}\right)$

for in the analysis. To keep the analyses simple, a stratified analysis approach to the data will be employed. ${ }^{16}$ For every patient in the study a four-fold table is constructed with site as the experimental unit of analysis. Subsequently, in attempting to combine information from all tables, three questions are investigated: ${ }^{15}$ 1) Is there evidence that the site-specific degree of association between the factor and the periodontal condition is consistent from patient to patient? 2) How large is the association as quantified by the odds ratio? and 3) Is the degree of site-specific association significant?

In a first step we can investigate the consistency of the site-specific responses across patients by using the BreslowDay statistic. ${ }^{16}$ The lower the $P$ value for this statistic, the more likely it is the data are inconsistent (i.e., heterogeneous). Since for this example the $P$ value of the BreslowDay statistic is $<0.001$, the test for the homogeneity of the effect is rejected and a careful reexamination of the data is indicated. A visual examination of the odds ratios of Table 1 reveals two different patient groups; one group in which exposure to factor A seems to be positively associated with the periodontal disease characteristic (those patients with odds ratios $>1$ ), suggesting that factor A may cause the disease characteristic, and another group of patients where factor A seems to be negatively associated with the disease characteristic (those patients with odds ratios < 1), suggesting that factor A may prevent the disease characteristic.

A first approach to this heterogeneity could be to assume that there is nothing peculiar about those inconsistencies and that the data should be analyzed as presented. Since the concept of homogeneity of effects is not a direct assumption of the Mantel-Haenszel procedures, it offers a valid analytic approach. ${ }^{17}$ The results of the analysis are shown in Table 2. The direction and extent of the relationship between factor $\mathrm{A}$ and the presence of the disease characteristic is quantified with the Mantel-Haenszel summary estimate of the odds ratio. The odds ratio of 2.4 suggests that the odds of exposure to factor A among diseased sites is 2.7 times larger than the odds of expose to factor $\mathrm{A}$ 
Table 2. Site-Specific Analysis Stratified on Patient

\begin{tabular}{lcc}
\hline $\mathrm{H}_{1}: \chi^{2}=26.87$ & DF $=7$ & Prob $<0.001$ \\
Summary Odds Ratio $=2.37$ & $95 \%$ Confidence Bounds $=\{1.38,4.08\}$ \\
$\mathrm{H}_{2}: \chi^{2}=9.74$ & DF $=1$ & Prob $=0.002$
\end{tabular}

Estimation of the association between factor $\mathrm{A}$ and the disease characteristic adjusted for the patient-effects. $\mathrm{H}_{1}$ tests the homogeneity of the responses across patients. For this example the test is highly significant indicating significant heterogeneity. $\mathrm{H}_{2}$ tests the significance of the overall association regardless of any heterogeneity, demonstrating a highly significant association between factor $\mathrm{A}$ and the disease characteristic.

Table 3. Site-Specific Analysis Stratified on Patient: Separation of Groups With Opposing Odds Ratios

\begin{tabular}{|c|c|c|}
\hline \multicolumn{3}{|c|}{ Males } \\
\hline $\mathrm{H}_{1}: \chi^{2}=0.377$ & $\overline{D F}=3$ & Prob $=0.945$ \\
\hline Summary Odds Ratio $=10.77$ & 95\% Confidence & Bounds $=\{4.40,26.34\}$ \\
\hline $\mathrm{H}_{2}: \chi^{2}=27.13$ & $\begin{array}{l}\text { DF }=1 \\
\text { Females }\end{array}$ & Prob $=0.001$ \\
\hline $\mathrm{H}_{1}: \chi^{2}=0.09$ & $\overline{\mathrm{DF}}=3$ & Prob $=0.993$ \\
\hline Summary Odds Ratio $=0.38$ & $95 \%$ Confidence & Bounds $=\{0.15,0.99\}$ \\
\hline $\mathrm{H}_{2}: \chi^{2}=3.92$ & $\mathrm{DF}=1$ & Prob $=0.048$ \\
\hline
\end{tabular}

$\mathrm{H}_{1}$ is the test for homogeneity and $\mathrm{H}_{2}$ is the test of significance for the overall association. Results of Table 2 are presented separately for males and females.

among non-diseased sites. The significance of this association is investigated with the Mantel-Haenszel $\chi^{2}$ statistic, which indicates a significant association between the presence of factor $\mathrm{A}$ and the disease characteristic at an alpha level of $0.05(P=0.002) .{ }^{18}$ The conclusion of this sitespecific analysis, which adjusted for patient effects by treating each as a stratum, indicated a significant association between the presence of factor $\mathrm{A}$ and the presence of the disease characteristic at periodontal sites. Although this approach is statistically correct, the results of such analyses may be considered improper and potentially misleading, since the patient-specific associations vary enough to indicate that there may be some other underlying effect modifier present.

A better approach would be to assume that there may be some biological explanation for the significant heterogeneity and to screen for patient-specific factors which may explain the inconsistency of the associations. Assuming for the sake of the example that the opposing trends of odds ratios coincided with sex, one simple approach would be to present the estimates separately for males and females. The strength, consistency, and significance of the association could be reassessed for the two subgroups as shown in Table 3. Within both sexes there is homogeneity of patient-effects $(P>0.95)$ and there exists significant, but opposing, associations between the factor and the disease characteristic. These findings could form a basis for future studies where the effect modification of sex on factor A could further be evaluated.

The previous analyses can be contrasted with the sitespecific analysis where the patient as a source of variation is ignored (Table 4). The odds of exposure to factor A are approximately equal for diseased and non-diseased sites.
Table 4. Site-specific Analysis Without Adjustment for the Patient Effects

\begin{tabular}{lcc}
\hline $\mathrm{H}: \chi^{2}=0.001$ & $\mathrm{DF}=1$ & Prob $=0.972$ \\
Crude Odds Ratio $=0.99$ & $95 \%$ Confidence Bounds $=\{0.63,1.56\}$
\end{tabular}

Analysis using pooled data. Note the switch-over bias and the absence of a significant association. $\mathrm{H}$ represents the hypothesis for testing significance of the crude (or unadjusted) association.

This association is of course insignificant $(P=0.99)$. The conclusion of this site-specific analysis, which does not adjust for the patient as a covariate, suggests that there is no association between the presence of a factor $\mathrm{A}$ and the disease characteristic.

Two issues for the analyses of site-specific data are illustrated in this example. First, adjustment for the patient may apparently yield different associations from unadjusted analyses (i.e., assuming that sites are independent). The switch-over bias seen in this example (from an odds ratio of 0.99 for the unadjusted analysis to an odds ratio of 2.4 for the adjusted analysis) is partly due to the fact that the patient-effect acted as a confounding factor. Apart from the site-specific factor under investigation, a paiient-specific factor was responsible for an effect. There seemed to be a trend for high prevalence of the exposure and a low risk for disease in the male group, and a low prevalence of exposure but a high risk for the disease in the female group. Hence, ignoring the confounding variable sex will make the association between the disease and the exposure appear less positive than it should be. (This assessment however may be irrelevant due to the highly significant interaction). Second, investigation of the homogeneity of the site-specific responses at a patient-level may play an important role for subsequent statistical analyses and for the interpretation of the data. Effect-modification of the patient-specific characteristics on the site-specific exposure variables can significantly affect the interpretation of the data and further analysis.

\section{DISCUSSION}

In many health research investigations, the relationship between a primary factor (e.g., a putative bacteriological organism) and the response variable (e.g., disease activity) is influenced by additional variables (e.g., the "patient" factor) which are also associated with the response variable. ${ }^{19}$ These additional variables can be labeled as intervening variables, confounding variables, effect modifiers, or covariables. Appropriate adjustments for these variables are necessary for a correct assessment of the relationship between site-specific variables. A wide variety of statistical models are available for the analysis of site-specific data. ${ }^{20-25}$ And, although the choice of the most suitable statistical technique may not always be straightforward, extreme solutions such as assuming that periodontal sites are independent units of observation should be avoided.

Adjustment for the patient as a covariate may have im- 
portant implications for the assessment of relationships between site-specific variables. Inconsistencies in the associations across patients can suggest the presence of additional causal mechanisms which may not have been considered in the study design. ${ }^{12}$ Consequently, it could form a contra-indication for pooling the site-specific data across patients and stimulate further inquiry on the host factor of those patients who responded differently. Occasionally, inconsistencies of the associations at a population level have generated new hypotheses that led to a better understanding of dental disease processes. For instance, inconsistent relationships between the plaque indices and both dental decay and periodontal disease severity provided an argument to support the specific-plaque hypothesis. ${ }^{26,27}$

Similarly, inconsistent responses at a patient level may be important in the study of periodontal disease. Several clinical trials, for instance, have indicated that for a given set of patients with an apparently similar periodontal disease history, disease activity seems to occur in a small subset of the studied subjects. ${ }^{28}$ Such findings would indicate that certain patient-factors may act as an effect modifier. If for analytic purposes the sites of all studied subjects are pooled without due consideration to the host-factor, associations between etiologic factors and disease activity, present in the small subgroup of patients, may be misrepresented. Sitespecific analyses which adjust for patient-effects may disclose characteristics of site-specific infections in periodontal disease entities which are clinically not self-evident, but which may become apparent through the investigation of the homogeneity of associations across patients. In general, analyses which take into account the sampling design from which the site-specific data originate may uncover unexpected departures from original hypothesis and isolate interesting patterns or features of the data.

Site-specific bacterial factors are most likely necessary, but not sufficient, causes of periodontal disease progression. Other components of sufficient causes are undoubtedly related to host factor characteristics such as the immune system. Consequently, the selection of an appropriate analysis for the site-specific data of a study is more than just a "statistical issue." Important concepts of causality such as consistency, strength, and specificity of an association may become obscured as a result of inappropriate analysis, and, more importantly, related topics such as effect modification due to the patient characteristics and/or confounding patient effects may become difficult to assess. It is concluded that sites can be used as the experimental unit of analysis, but that the sampling design from which the site-specific data originated should not be ignored.

\section{REFERENCES}

1. Socransky SS, Haffajee AD, Smith GLF, Dzinck JL. Difficulties encountered in the search for the etiologic agents of destructive periodontal diseases. J Clin Periodontol 1987; 14:588.
2. Haffajee AD, Socransky SS. Attachment level changes in destructive periodontal diseases. J Clin Periodontol 1986; 13:461.

3. Goodson JM. Clinical measurement of periodontitis. J Clin Periodontol 1986; 13:466.

4. Fidler V. Letter to the editor. J Clin Periodontol 1984; 11:138.

5. Blomqvist $\mathrm{N}$. On the choice of the computational unit in statistical analysis of unbalanced clinical trials. J Clin Periodontol 1987; 14:519.

6. Fleiss JL, Wallenstein S, Chilton NW, Goodson JM. A re-examination of within-mouth correlations of attachment level and of change in attachment level. $J$ Clin Periodontol 1988; 15:411.

7. Laster LL. The effect of subsampling sites within a patient. $J$ Periodont Res 1985; 20:91.

8. Zimmerman SO. Discussion: Attachment level changes in destructive periodontal disease. J Clin Periodontol 1986; 13:473.

9. Hill AB. The environment and disease: Association or causation? Proceedings of the Royal Society of Medicine. 1965; 58:295.

10. Rothman KJ. Causal inference in epidimiology. Modern Epidimiology. Boston/Toronto: Little, Brown and Company; 1986:7-21.

11. Mantel N, Brown C, Byar DP. Test for homogeneity of effect in an epidemiologic investigation. Am J Epid 1977; 106(2):125.

12. Kleinbaum DG, Kupper LL, Morgenstern H. Epidemiologic Research. New York: Van Nostrand Reinhold Book; 1982; 33.

13. Holland PW. Statistics and causal inference. JASA 1986; 12:945.

14. Kleinbaum DG, Kupper LL, Morgenstern H. Epidemiologic Research. New York: Van Nostrand Reinhold Book; 1982:243-265.

15. Fleiss JL. Statistical Methods for Rates and Proportions. 2nd ed. New York: John Wiley \& Sons; 1981:160-187.

16. Breslow NE, Day NE. Statistical Methods in Cancer Research. Volume 1: The Analysis of Case-Control Studies. IARC Scientific Publications 1980:142.

17. Landis JR, Heyman ER, Koch GG. Average partial association in three-way contingency tables: A review and discussion of alternative tests. Int Stat Rev 1978;46:237.

18. Mantel N, Haenszel W. Statistical aspects of the analysis of data from retrospective studies of disease. $J$ Natl Cancer Inst 1959; 22:719.

19. Landis RJ. The Analysis of Categorical Data: Applications to the Biomedical and Health Sciences. Ann Arbor: University of Michigan; 1987:219.

20. DeRouen TA. Biostatistical and methodological issues in demonstrating efficacy of therapeutic agents for periodontal diseases. $J$ Dent Res 1989; 68:1161.

21. Donner A, Banting DW. Statistical analysis of site-specific data in dental studies. J Dent Res 1988; 67:1392.

22. Donner A, Banting DW. Adjustment of frequently used chi-square procedures for the effect of site-to-site dependencies in the analysis of dental data. $J$ Dent Res 1989; 68:1350.

23. Brier SS. Analysis of contingency tables under cluster sampling. Biometrika 1980; 67:591.

24. Sterne JAC, Johnson NW, Wilton JMA, Joysten-Bechal S, Smales FC. Variance components analysis of data from periodontal research. J Periodont Res 1988; 23:148.

25. Hujoel PP, Loesche WJ, Moulton LH. Estimation of sensitivity and specificity of site-specific diagnostic tests. $J$ Periodont Res (Accepted for publication).

26. Loesche WJ. Chemotherapy of dental plaque infections. Oral Sci Rev 1976; 9:63

27. Loesche WJ. Role of Streptococcus mutans in dental decay. Microbiol Rev 1986; 50(4):353.

28. Listgarten MA. Pathogenesis of periodontitis. J Clin Periodontol 1986; 13:418.

Send reprint requests to: Dr. Philippe P. Hujoel, Department of 'Dental Public Health Sciences, University of Washington, SM-35, Seattle, WA 98195.

Accepted for publication December 22, 1989. 